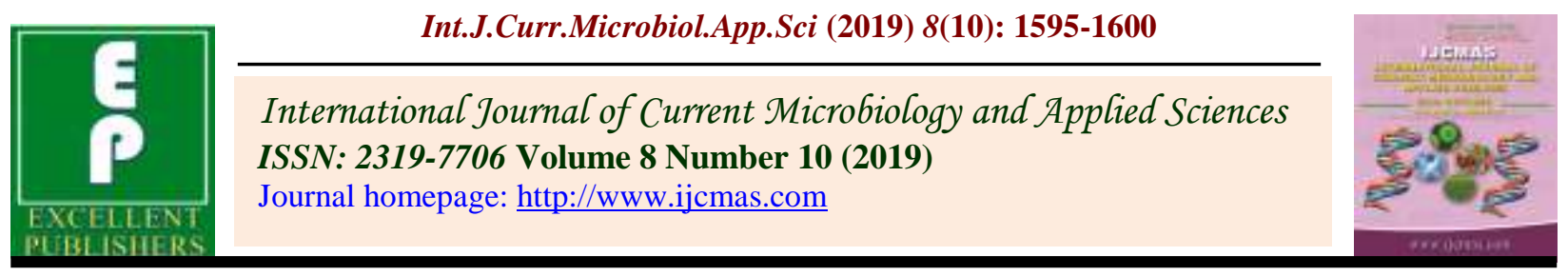

Original Research Article https://doi.org/10.20546/ijcmas.2019.810.186

\title{
Serodiagnosis of Rickettsial Infections among Febrile Paediatric Patients in a Tertiary Care Hospital
}

\author{
Vikhyath P. Raj, D. R. Gayathri Devi* and T. Sandeep \\ Department of Microbiology, M. S. Ramaiah Medical College, Bangalore, India \\ *Corresponding author
}

\section{A B S T R A C T}

Keywords

PUO, Rickettsia,

Weil-Felix test

Article Info

Accepted:

12 September 2019

Available Online:

10 October 2019
Rickettsial disease is one among the most common re-emerging disease and untreated cases having fatality rates as high as $30-35 \%$ and since it is a cause of fever of unknown origin(FUO) it needs to be differentiated from other febrile illnesses. The present study was performed at a tertiary care Centre in Bangalore after taking Ethical Clearance from the Institution's Ethical Committee. All 101 Paediatric patients admitted in the Paediatric department of M. S. Ramaiah Hospitals were included in the study in the year 2015 . The study revealed $5.95 \%$ of the suspected cases to be positive for Weil Felix test with significant titers.

\section{Introduction}

Rickettsial disease is one among the most common re-emerging infections as well as important causes of Fever of Unknown Origin (FUO). They are generally incapacitating in nature and very difficult to diagnose $(1,2)$.

Family Rickettsiaceae comprises a group of microorganisms that occupy a position between viruses and bacteria. The Family Rickettsiaceae comprises of three genera namely Rickettsia, Orentia and Ehrilichia. Structurally these organisms are small, nonflagellate, Gram negative cocco-bacilli. They are transmitted by arthropods and are obligate intracellular parasites. They are primary parasites of arthropods like lice, fleas, mites and ticks. These arthropods further act as vectors and spread the infection (3).

Humans are accidental hosts and acquire infection from the above mentioned infected arthropod vectors or when exposed to an animal reservoir host. On entering the host, they invade and lyse their target cells after the organisms multiply and accumulate in large numbers in the same or escape the cells, damaging its membrane and causing an influx of water. 
The target cells are vascular endothelial cells, reticulo-endothelial cells and blood cells (1). They are not transmissible from person to person in general except by blood transfusion or organ transplantation.

Their incubation period varies from 2-21 days and is present in a mild or severe form. Clinical observations include fever, headache, myalgia, rashes, eschar, abdominal pain, cough, dizziness, disorientation and vasculitis $(4,5)$.

The complications of these diseases result predominantly due to the infection of endothelial cells that determines the development of multi-systemic small vessel vasculitis, which may affect lungs (interstitial pneumonitis), heart (myopericarditis), skin (rash), central nervous system (meningoencephalitis), liver, and kidneys (6).

The diagnosis of Rickettsial infections is difficult as it gives very high titre values at the end of the 2 nd week but declines during convalescence, however if diagnosed they are often easily treated.

Diagnosis is done by a serological test called Weil-Felix test. This test is a heterophile agglutination test which uses antigens from the standard strains of Proteus vulgaris \& Proteus mirabilis.

Weil-Felix test is not a very sensitive test in diagnosis of scrub typhus but due to the lack of availability of definitive tests in India it can be a helpful tool when used and interpreted in the correct clinical context (4).

The main aim and objectives of the study includes, to study the proportion of Rickettsial infections among children with fever for more than 7 days or Fever with rash using WeilFelix test.

\section{Materials and Methods}

The present study was performed at a tertiary care Centre in Bangalore after taking Ethical Clearance from the Institution's Ethical Committee.

A total of 101 Paediatric patients admitted in the Paediatric department of M. S. Ramaiah Hospitals were included in the study in the year 2015. An Informed Consent from the parent or guardian of the subject, were included in the study.

In the present study the inclusion criteria followed was Children between the ages of 2 and 18 years coupled with fever of more than 7 days with or without rash. While the exclusion criteria was such of those children diagnosed clinically and serologically with viral (measles, dengue), bacterial (typhoid), protozoan (malaria) and collagen vascular diseases.

\section{Method of data collection}

The detailed clinical history along with their demographic profile was obtained from the parents or guardians of 101 patients.

3 - $5 \mathrm{ml}$ of blood was collected in a vacutainer without anticoagulant from the patient under aseptic precautions from the median cubital vein.

The vacutainers were centrifuged, serum was separated and stored in Eppendorf tubes at $20^{0} \mathrm{C}$ till the Weil Felix test was performed.

\section{Weil-Felix test}

The sharing of antigens between rickettsia and proteus forms the principle of the heterophile antibody test. It demonstrates agglutinins to Proteus vulgaris strain OX 19, OX 2 and OX K. 
The test was performed using commercially available antigen kit supplied by Omega.

The test is a tube agglutination test and a set 7 tubes were used for 1 antigen.

$1.9 \mathrm{ml}$ of normal saline in the first tube and $1 \mathrm{ml}$ normal saline to the remaining 6 test tubes

Add $0.1 \mathrm{ml}$ of serum to the first tube and then transfer $1 \mathrm{ml}$ of the well mixed diluted sample to the next tube

Perform this transfer similarly to other test tubes, discarding $1 \mathrm{ml}$ in the end after the 7 th tube to obtain sample dilutions ranging from $1: 20$ to $1: 1280$

One drop (30-50 $\mu 1)$ of specific antigen OX 2, OX 19 and OX K are added to separate sets of tubes and thoroughly mixed

The test tubes are incubated overnight at $37^{0} \mathrm{C}$ and observed for agglutination

The agglutination is a coarse granular mat like formation seen at the bottom of the tube.

Settling of the antigens to the bottom of the tube - Button formation, is regarded as negative test

The highest dilution of the serum giving a visible agglutination is taken as the titre

Any titre $\geq 1: 160$ is considered to be positive and diagnostic of rickettsial infection.

\section{Statistical methods}

Proportion of rickettsial infections was analysed and summarized in terms of percentage Chi square test was used to compare rickettsial infections between different ages and sex.

\section{Results and Discussion}

Of all the patients admitted in the pediatrics department, 101 patients satisfied the inclusion criteria and gave consent to be included in the study. Weil -Felix test was positive in six patients out of 101 (5.95\%) clinically suspected for rickettsial fever.

Among six patients one was presented with fever and rash where as others showed only fever (Table 1) The number of suspected cases were more in 11-15 years age followed by 610 years age (Table 2). There were no significance differences between the sex distribution (Table 3).

Among all the patients admitted to the paediatric department, a total number of 101 patients have satisfied the inclusion criteria and have given consent to be a part of this study. Inclusion criteria being considered in the study were - Children between the ages of 2 and 18 years (paediatric age group), Fever of more than 7 days with or without rash. Similar criteria were used in other studies involving rickettsial infections (2).

The patient demographic characteristics were taken in order avoid selection bias. In this study there was similar representation in both the sexes (Graph 2). The age distribution was uniform throughout the paediatric age group (Graph 1). Therefore the study population is homogenous and unbiased. Weil Felix test is heterophile agglutination test which uses antigens from the standard strains of Proteus vulgaris \& Proteus mirabilis. Issac and his colleagues found a sensitivity for OX-K was $30 \%$ at a titre breakpoint of 1:80, but the specificity and positive predictive value were 100\%.(9) Micro-immunofluorescence gives more accurate titres for the diagnosis of Rickettsial fevers but considering the cost and practical issue related to performing the test, Weil Felix was chosen for this study. 
Table.1 Overview of Weil - Felix test results

\begin{tabular}{|c|c|c|}
\hline Result & Frequency & Percent \\
\hline Negative & 95 & 94.05 \\
\hline Positive & 6 & 5.95 \\
\hline Total & 101 & 100.0 \\
\hline
\end{tabular}

\begin{tabular}{|c|c|c|}
\hline & Fever of 7 days & Fever with rash \\
\hline Negative & 90 & 5 \\
\hline Positive & 5 & 1 \\
\hline
\end{tabular}

Table.2 Age distribution

\begin{tabular}{|c|c|c|c|}
\hline \multirow{2}{*}{ Age Group } & \multicolumn{2}{|c|}{ Weil-Felix result } & Total \\
\cline { 2 - 3 } & Negative & Positive & \\
\hline $2-5 \mathrm{yr}$ & 19 & 0 & 19 \\
\hline $6-10 \mathrm{yr}$ & 21 & 2 & 23 \\
\hline $11-15 \mathrm{yr}$ & 38 & 2 & 40 \\
\hline $16-18 \mathrm{yr}$ & 17 & 2 & 19 \\
\hline Total & 95 & 6 & 101 \\
\hline
\end{tabular}

$\mathrm{p}$ value $=0.51$

Table.3 Sex Distribution

\begin{tabular}{|c|c|c|c|}
\hline & \multicolumn{2}{|c|}{ Weil-Felix result } & \multirow{2}{*}{ Total } \\
\cline { 1 - 3 } Sex & Negative & Positive & \\
\hline Female & 42 & 3 & 45 \\
\hline Male & 53 & 3 & 56 \\
\hline Total & 95 & 6 & 101 \\
\hline p value $=0.78$ & & & \\
\hline
\end{tabular}

Graph.1 Age distribution

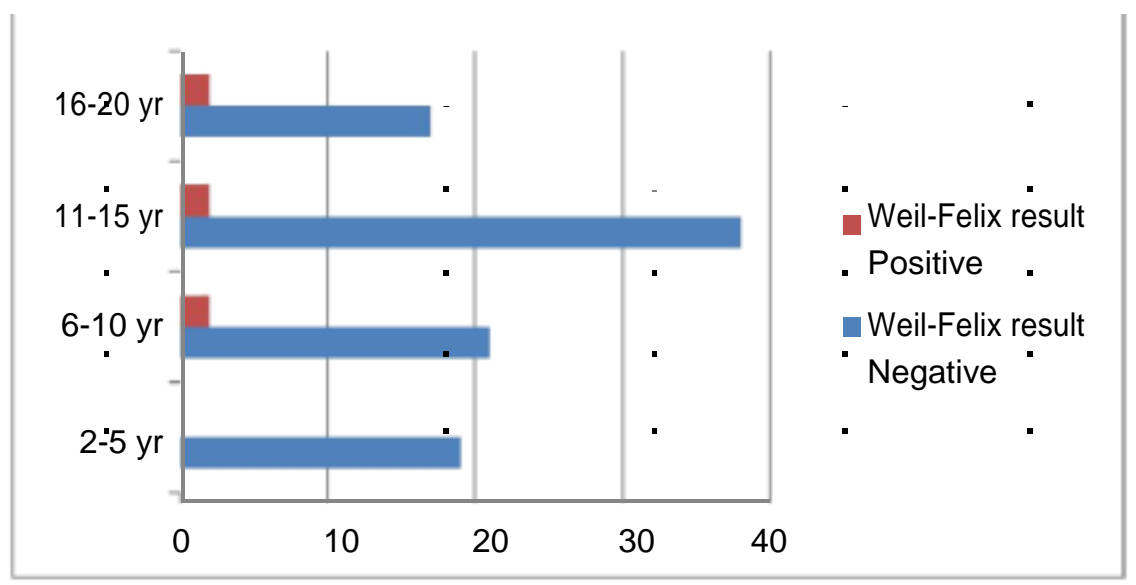


Graph.2 Sex distribution

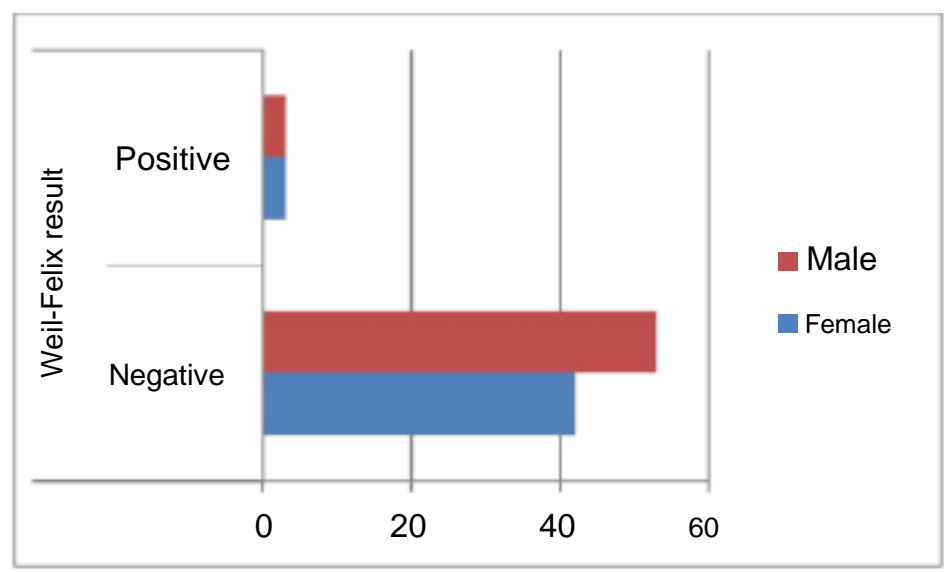

Seropositivity was observed as $\underline{5.95 \%}$ of a study population of 101 patients.

In this study it was found that $5.95 \%$ of the suspected cases had Weil Felix test positive with significant titres. The cut off chosen for the study is $\geq 1: 160$. A study by Veena Mittal, et al., suggests this cut off based on the endemic pattern (2).

The sensitivity and specificity varies (increases and decreases respectively) when a lower cut off titre is chosen as more patients would have antibodies present which may not co-relate to the clinical symptoms giving false positives. (9)

Agglutination was seen in $5.95 \%$ of samples of which 4 were positive for OX2 suggesting endemic typhus and 2 were positive for OXK suggesting scrub typhus. Other studies give a prevalence rate ranging from $9.2 \%$ to $33.3 \%$ $(7,2)$. Therefore the $5.95 \%$ positivity obtained from this study conducted in a tertiary care setup suggests a lesser rate of incidence of Rickettsial fevers.

Another reason for the lower rate of positivity could be because of the patients taking treatment at a peripheral center with broad spectrum antibiotics rather than presenting themselves in a tertiary care center.

The $\mathrm{p}$ values obtained from Tables 2 and 3, for the age and sex distribution are 0.51 and 0.78 respectively, which are not significant. Also indicating that there is no co-relation of the age and sex of the individual with the disease. The confidence interval obtained for this study is $1.24-10.66$.

This study gives us a seropositivity of $5.95 \%$ of a sample size of 101 patients of the paediatric age group.

A further study if conducted at a larger scale involving both the adult and paediatric population considering the patients recent history of travel and their demographic details would give us a clearer picture of the endemicity and prevalence of these rickettsial diseases

\section{Financial support and Sponsorship}

I C M R-STS Project

\section{References}

Bernabeu-Wittel M, Segura-Porta F, "Rickettsioses", Enferm Infecc Microbiol Clin. 2005 Mar;23(3):16372.

Isaac R, Varghese GM, Mathai E, et al., 
"Scrub Typhus: Prevalence and Diagnostic Issues in Rural Southern India." Clin Infect Dis 2004; 39:13956.

Jayaram Paniker CK. Ananthanarayan and Paniker's Textbook of Microbiology, 9th edition, University Press Pvt Ltd; 2013; 405-414.

Kamarasu K, Malathi M, et al., "Serological evidence for wide distribution of spotted fevers and typhus fevers in Tamil Nadu ", Indian J Med Res, 2007 Aug; 126(2):128-30

Mahajan SK, Kashyap R, et al., "Relevance of Weil-Felix test in the diagnoses of scrub typhus in India", $J$ Assoc Physicians India, 2006; 4: 619-21.

Narendra Rathi and Akanksha Rathi,
"Rickettsial Infections", Indian Pediatrics, 2010 Feb, vol 47, 157164.

Prabhakaran A, Lal S, Biswas S, Vinoth S, Asraf AS, Mittal V Serological study of rickettsial diseases in human and rodent population in Chittoor dist. (A.P.). J Commun Dis. 2010 Sep; 42(3):209-13.

Somashekar HR, Prabhakar DM, et al., "Magnitude and features of scrub typhus and spotted fever in children in India", J Trop Pediatr, 2006; 52: 229.

Veena Mittal, Naveeen Gupta, et al., "Serological evidence of rickettsial infections in Delhi", Indian J Med Res 135, April 2012, pp 538-541.

\section{How to cite this article:}

Vikhyath P. Raj, D. R. Gayathri devi and Sandeep, T. 2019. Serodiagnosis of Rickettsial Infections among Febrile Paediatric Patients in a Tertiary Care Hospital. Int.J.Curr.Microbiol.App.Sci. 8(10): 1595-1600. doi: https://doi.org/10.20546/ijcmas.2019.810.186 